\title{
Descompresión orbitaria endoscópica transnasal en orbitopatía por enfermedad de Graves
}

\author{
Martín Alonso Pinzón Navarro ${ }^{1}$ Perla Villamor Rojas ${ }^{2}$
}

\author{
${ }^{1}$ Otorrinolaringólogo, docente adscrito, coordinador de Residentes, \\ Servicio de Otorrinolaringología, Hospital de San José. Fundación \\ Universitaria de Ciencias de la Salud, Bogotá, Colombia. \\ ${ }^{2}$ Residente de Otorrinolaringología, II año, Fundación \\ Universitaria de Ciencias de la Salud - Hospital de San José. \\ Bogotá, Colombia.
}

Correo electrónico: perlviro@hotmail.com, pvillamayor@ fucsalud.edu.co

\section{Resumen}

Introducción: La orbitopatía distiroidea ocasiona un aumento del volumen orbitario, exoftalmos y síntomas oculares severos como pérdida de la agudeza visual, diplopía y compromiso del nervio óptico. Los corticoesteroides y la radioterapia han sido usados para su tratamiento pero con efectos secundarios indeseables. La cirugía se puede considerar una de las mejores opciones de manejo.

Objetivo: El objetivo del estudio es describir los resultados quirúrgicos de los pacientes con orbitopatía por enfermedad de Graves sometidos a descompresión endoscópica transnasal de órbita en el Hospital de San José en Bogotá, Colombia, entre el año 2009 y 2014.

Métodos: Se realizó un estudio descriptivo longitudinal de serie de casos. Se analizaron los datos con base en cambios posoperatorios de agudeza visual, campimetría y exoftalmometría, así como complicaciones posoperatorias.

Resultados: Se analizaron 32 órbitas de 16 pacientes. El $83,3 \%$ de los pacientes presentaba campimetrías pre y posoperatorias normales, excepto por defectos leves secundarios a ptosis palpebral. El 87,5\% presentó agudeza visual preoperatoria peor o igual a $20 / 50$, con mejoría de la agudeza visual en el posoperatorio en el $100 \%$ de los casos. La exoftalmometría preoperatoria tuvo una mediana de $24 \mathrm{~mm}$ (RIQ: 21,8-27) (DS: $2,77)$, mientras que la posquirúrgica presentó una mediana de 1,25 mm (RIQ:16-21) (DS: 2,99), la cual se considera un estudio dentro de límites normales. La única complicación reportada fue la presencia de diplopía en 4 de los 16 pacientes (25\%), de los cuales una fue transitoria y 3 persistieron durante todo el seguimiento $(18,75 \%)$.

Conclusiones: Existen limitaciones en el estudio al ser retrospectivo descriptivo; sin embargo, los datos obtenidos muestran a la descompresión orbitaria transnasal para orbitopatía de Graves como una intervención segura y eficaz.

\begin{abstract}
Introduction: Dysthyroid orbitopathy causes an increase in orbital volume, exophthalmos and severe ocular symptoms such as loss of visual acuity, diplopia, and optic nerve involvement. Corticosteroids and radiotherapy have been used for treatment but with undesirable side effects. The surgery can be considered one of the best treatment options.
\end{abstract}

Objective: The aim of the study was to describe the surgical outcomes of patients with Graves' orbitopathy, treated with transnasal endoscopic orbital decompression in Hospital de San José in Bogotá, Colombia, between 2009 and 2014.

Methods: A descriptive longitudinal case series study was conducted. Data were analyzed taking into account postsurgical changes of visual acuity, exophthalmometry and campimetry, also postoperative complications.

Results: 32 orbits of 16 patients were analyzed. 83.3\% of patients had pre and postoperative normal campimetries except slight defects, due to palpebral ptosis. $87.5 \%$ had preoperative visual acuity worse or equal than 20/50, with improved visual acuity postoperatively in $100 \%$ of cases. The preoperative exophthalmometry $24 \mathrm{~mm}$ had a median (IQR 21.8-27) (SD: 2.77), while the postoperative median was $18.25 \mathrm{~mm}$ (IQR 16-21) (SD: 2.99), which is considered a normal study. The only complication reported was the presence of diplopia in 4 of the 16 patients (25\%), of which 1 was transient and 3 persisted throughout follow-up (18.75\%).

Conclusions: There are limitations on the study due to its retrospective nature, but the data showed the transnasal orbital decompression for Graves orbitopathy as a safe and effective intervention.

\section{Introducción}

La enfermedad de Graves es una enfermedad autoinmune, multiorgánica, de fisiopatología compleja que afecta principalmente a la glándula tiroides y la órbita. Es una causa común de hipertiroidismo cuando las inmunoglobulinas estimulantes de la tiroides, producidas por los linfocitos $B$, se unen al receptor de la hormona estimulante de la tiroides. 
El mecanismo exacto de la enfermedad ocular relacionada con la enfermedad tiroidea es desconocido y se cree que los fibroblastos en los tejidos orbitales pueden expresar el receptor de la hormona estimulante de la tiroides (TSHR). Posteriormente se presenta una acumulación de linfocitos $\mathrm{T}$ sensibilizados a antígenos tiroideos compartidos con los contenidos orbitarios, lo que conduce a anomalías inmunológicas que causan infiltración de la grasa orbital y los músculos extraoculares con células inflamatorias y complejos inmunes. Por último, esta infiltración grasa y muscular termina por ocasionar un aumento en el volumen orbitario extraocular y en la presión intraocular ${ }^{(1)}$.

Aproximadamente el $50 \%$ de los pacientes con enfermedad de Graves desarrollará manifestaciones orbitarias, siendo más común en las mujeres. Sin embargo, cuando la enfermedad está presente en los hombres es generalmente más severa y agresiva. Los fumadores desarrollan orbitopatía distiroidea con más frecuencia y su enfermedad es más grave en la fase activa, con posterior fibrosis de los tejidos afectados ${ }^{(2,3)}$.

La orbitopatía distiroidea es la manifestación extratiroidea más común de la enfermedad de Graves ${ }^{(4,5)}$, la cual consiste en un proceso autoinmune que genera un incremento en el volumen del tejido orbitario. Aunque se han informado casos de resolución espontánea, con frecuencia el dolor, la queratitis por exposición, la diplopía, la neuropatía óptica y la estética son indicaciones para iniciar un tratamiento.

Se consideran factores de riesgo reconocidos para desarrollar orbitopatía distiroidea: los antecedentes familiares, la edad (20 a 50 años), el sexo femenino, el tabaquismo, un tratamiento previo con yodo radioactivo y la disfunción tiroidea. Aunque varios de los factores de riesgo como la genética, la edad y el sexo, no se pueden modificar, existen factores de riesgo modificables para la disminución de la presentación y progresión de la orbitopatía distiroidea, como el tabaquismo y la exposición a yodo radioactivo ${ }^{(3)}$.

Las manifestaciones clínicas de la orbitopatía distiroidea van desde síntomas leves, como epífora, fotofobia e inyección conjuntival, hasta síntomas graves, como proptosis significativa, diplopía, queratopatía por exposición, y pérdida de la visión por neuropatía óptica(6).

El curso clínico de la orbitopatía de Graves se puede dividir en fases agudas y crónicas. La fase aguda se caracteriza por la inflamación activa que va de 6 a 18 meses, mientras que la fase crónica se caracteriza por fibrosis y representa la fase preferida para la intervención quirúrgica(7,8).

Imaginológicamente no es posible medir de forma exacta el volumen completo de los músculos extraoculares. Sin embargo, el uso de tomografía computarizada o resonancia magnética permite calcular los volúmenes musculares de forma $\operatorname{aproximada}^{(9)}$.

El diagnóstico diferencial incluye enfermedades y tumores inflamatorios, de los cuales el pseudotumor orbitario, la mio- sitis ocular y el linfoma orbital son los más importantes. Los patrones específicos: localización, participación de estructuras orbitales y los cambios de señal se pueden diferenciar por resonancia magnética ${ }^{(10)}$.

La orbitopatía distiroidea es a menudo un trastorno autolimitado y, en la mayoría de los casos, se produce la resolución espontánea. Las medidas locales, como la lubricación, cinta en los párpados y uso de parches para los pacientes con resequedad y diplopía representan enfoques iniciales del tratamiento conservador. Sólo una minoría de los pacientes con enfermedad severa (3 a 5\%) requieren tratamientos invasivos ${ }^{(11)}$.

Los tratamientos más agresivos incluyen los corticoesteroides sistémicos y el uso de la radiación orbitaria. Los corticoesteroides sistémicos se usan con frecuencia para tratar la orbitopatía; sin embargo, conllevan usualmente efectos secundarios. La radiación, aunque se usa actualmente, no está respaldada por un ensayo clínico controlado aleatorio que demuestre su seguridad y eficacia. Por lo anterior, la cirugía sigue siendo una de las mejores opciones en el manejo de la orbitopatía por Graves ${ }^{(12)}$.

Desde principios de 1990, la descompresión orbitaria endoscópica se ha convertido en el tratamiento quirúrgico de elección para los pacientes con orbitopatía distiroidea. En comparación con los enfoques externos para la descompresión orbitaria, la técnica endoscópica proporciona una mejor visualización de las regiones anatómicas críticas, incluidas la base del cráneo y el vértice de la órbita, evitando incisiones faciales o intraorales ${ }^{(13)}$.

La descompresión de órbita consiste en una exposición de la totalidad de la pared medial orbitaria (lámina papirácea)

(figura 1), la cual se fractura inicialmente sin violar la capa de periostio orbital (periórbita) que encierra el contenido orbital, posterior a la eliminación del hueso se incide la periórbita a lo largo de toda su longitud horizontal de posterior a anterior por medio de tres incisiones paralelas aproximadamente a $1 \mathrm{~cm}$ de distancia, lo cual da lugar a un prolapso de la grasa orbitaria y la consiguiente disminución de la proptosis ${ }^{(14)}$ (figura 2).

La orbitopatía por Graves estable e inactiva puede ser tratada con éxito mediante cirugía, ya sea la descompresión orbitaria con el fin de reducir la proptosis, la resección del músculo para corregir la diplopía o, finalmente, la blefaroplastia para corregir la ptosis secundaria ${ }^{(15)}$.

\section{Materiales y métodos}

Se realizó un estudio descriptivo longitudinal de serie de casos con pacientes con diagnóstico de orbitopatía por enfermedad de Graves, pacientes con diagnóstico establecido de hipertiroidismo y exoftalmos, tratados con descompresión endoscópica nasal orbitaria, método estandarizado y realizado por el mismo cirujano en todos los casos, en el servicio de 
Figura 1. Anatomía orbitaria normal

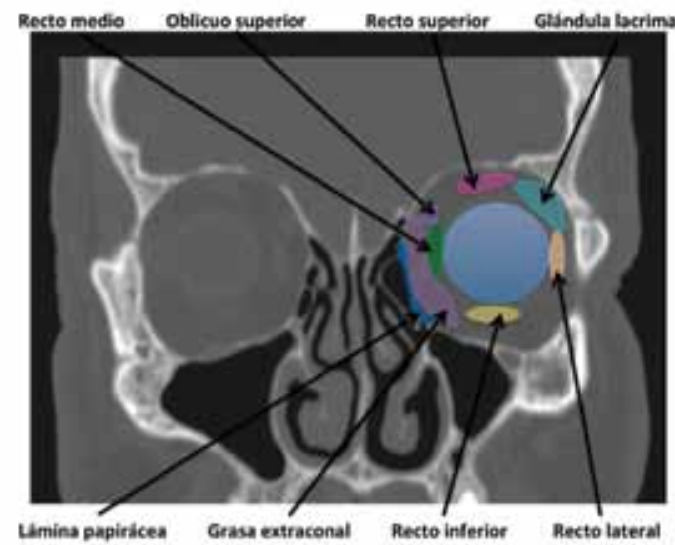

A. Corte axial de tomografía computarizada (TC) de senos paranasales con superposiciones en color que muestran la órbita dividida en sus espacios intraoculares y extraoculares y sus relaciones con la lámina papirácea, celdillas etmoidales y cavidad nasal.

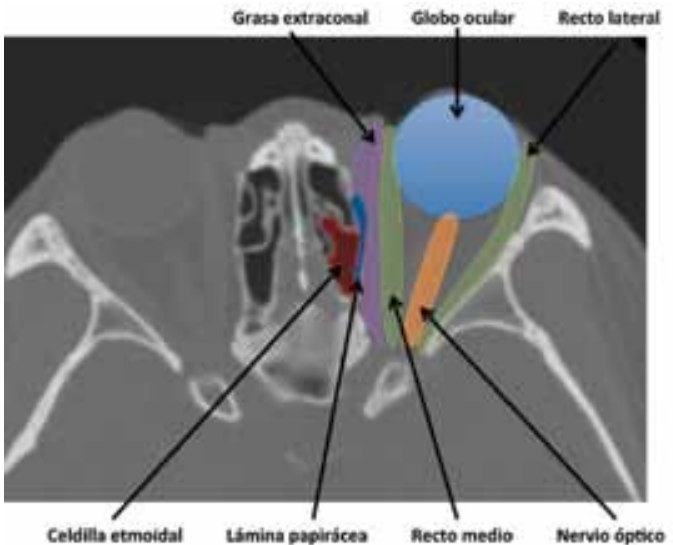

B. Corte coronal de TC de senos paranasales con superposiciones en color que muestran la configuración de los músculos extraoculares, y la glándula lagrimal, así como sus relaciones con la lámina papirácea y la cavidad nasal.
Figura 2. Vista endoscópica fosa nasal izquierda

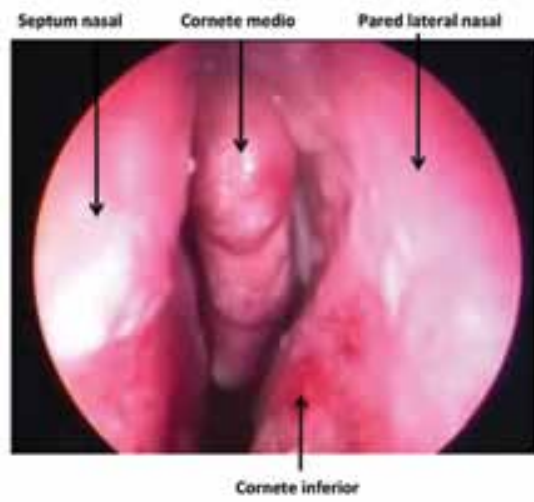

A. Visión preoperatoria.

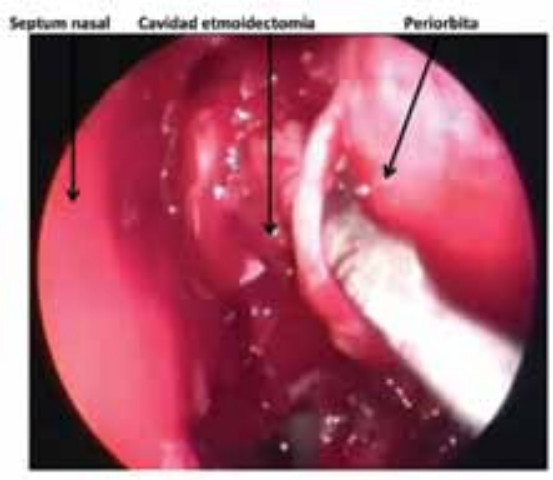

C. Mediante un bisturí en hoz se abre la periórbita cuidadosamente sin lesionar el recto medio (evitando riesgo de diplopía).

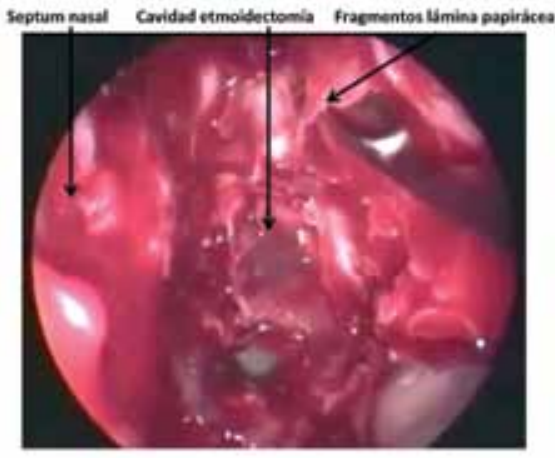

B. Una cureta es utilizada para fracturar la lámina papirácea y comenzar la descompresión ósea.

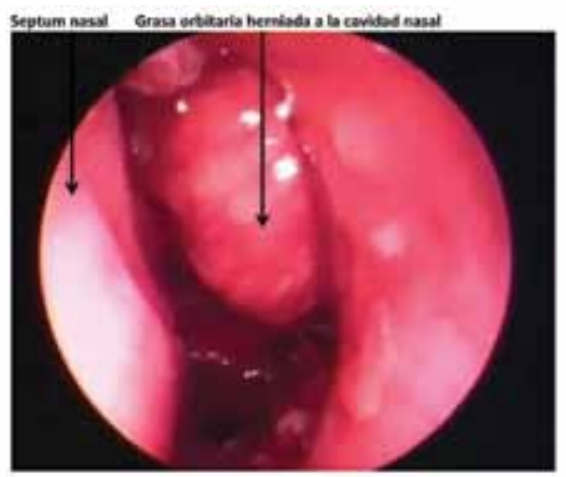

D. Grasa orbitaria herniada hacia la cavidad nasal. otorrinolaringología del Hospital de San José, entre los años 2009 y 2014. Se realizó una revisión de historias clínicas de los pacientes tratados, de donde se obtuvieron los datos para el estudio.

El abordaje quirúrgico se realizó en todos los pacientes, de la siguiente forma: bajo visión endoscópica transnasal, infiltración de la pared lateral nasal y del cornete medio con lidocaína al $1 \%$ con epinefrina, medialización del cornete medio, antrostomía maxilar, identificación del receso nasofrontal, logrando adecuada permeabilización del mismo, etmoidectomía anterior y posterior, identificación de lámina papirácea para su posterior resección, identificando periórbita, la cual se incide con bisturí frío, permitiendo herniación de la grasa periorbitaria hacia cavidad de etmoidectomía.

Se excluyeron pacientes que no completaron el seguimiento posoperatorio mínimo de un mes. Se hizo un seguimiento hasta el último control posoperatorio, teniendo en cuenta las variables sociodemográficas como edad y sexo, agudeza visual, campimetría y exoftalmometría obtenidas por un especialista en ofatlmología de forma estandarizada, tomografía computarizada y/o ecografía orbitaria. 
Las variables cuantitativas se exploraron con pruebas estadísticas para determinar su distribución y presentarlas con medidas de tendencia central y dispersión. Las variables cualitativas se presentaron con frecuencias absolutas y relativas. La información se analizó en Stata 12.0.

\section{Resultados}

Se analizaron 16 pacientes, de los cuales 12 eran mujeres (75\%), 4 hombres (25\%), la mediana de edad fue de 44 años (RIQ: 37-54; DS: 11,15) al momento de la cirugía (tabla 1). Del total de los pacientes, sólo 2 (12,5\%) eran fumadores o tenían antecedente de tabaquismo, y el 100\% presentó compromiso de ambas órbitas, para un total de 32 órbitas tratadas.

Todos los pacientes se sometieron a valoración endocrinológica y oftalmológica, todos los pacientes sometidos a cirugía contaban con la estabilización de su patología de base, verificada por pruebas de función tiroidea dentro de límites normales.

Todos los pacientes se sometieron a descompresión endoscópica transnasal de órbita como se describió en la sección materiales y métodos.

Todos los pacientes contaron con imágenes prequirúrgicas, tomografía computarizada de órbita en el 75\% y ecografía de órbita en el 25\% restante. En ambos estudios, el 100\% de los pacientes presentó cambios orbitarios dados por aumento de grados leve a severo del contenido y grasa intraorbitaria, aumento de los músculos rectos superiores e internos ocasionando exostosis de los globos oculares.

De las 32 órbitas, 24 contaban con campimetría preoperatoria, de las cuales $20(83,3 \%)$ presentaron un estudio dentro de límites normales, mientras que los restantes tan solo mostraban defectos leves correspondientes a alteración de tipo palpebral, todos los pacientes contaron con campimetrías posoperatorias dentro de límites normales, excepto 4 (12,5\%) que persistieron con defectos secundarios a ptosis palpebral.

En cuanto a la agudeza visual, 28 órbitas $(87,5 \%)$ presentaron agudeza visual preoperatoria peor o igual a $20 / 50$, reduciéndose el número a tan solo 2 órbitas $(6,25 \%)$ en el posoperatorio con agudeza visual peor o igual a 20/50, y no se presentó ningún caso de empeoramiento o casos de alteración de agudeza visual de novo en el posoperatorio.

De las dos órbitas con neuropatía óptica preoperatoria, el $100 \%$ presentó resolución completa, y no se presentó ningún caso de neuropatía óptica de novo en el posoperatorio.

La exoftalmometría prequirúrgica presentó una mediana de $24 \mathrm{~mm}$ (RIQ: 21,8-27) (DS: 2,77), comparada con una exoftalmometría posquirúrgica con una mediana de $18,25 \mathrm{~mm}$ (RIQ:16-21) (DS: 2.99), la cual se considera un estudio dentro de límites normales (figura 3).

En los estudios posoperatorios se identificó como única complicación reportada la presencia de diplopía en 4 de los 16 pacientes (25\%), de los cuales una fue transitoria y tres persistieron durante todo el seguimiento $(18,75 \%)$. Ningún paciente presentó estrabismo o alteración de la movilidad ocular posoperatoria.
Tabla 1. Características sociodemográficas y clínicas de los pacientes y órbitas en estudio

\begin{tabular}{|c|c|}
\hline \multicolumn{2}{|l|}{ Sexo n (\%) } \\
\hline Masculino & $4(25)$ \\
\hline Femenino & $12(75)$ \\
\hline Edad (mediana, años, (RIQ)(DS) & $44(37-54)(11,15)$ \\
\hline \multicolumn{2}{|l|}{ Fumador $\mathrm{n}(\%)$} \\
\hline Sí & $2(12,5)$ \\
\hline No & $14(87,5)$ \\
\hline \multicolumn{2}{|l|}{ Imágenes radiológicas preoperatorias n (\%) } \\
\hline Tomografía computarizada de órbita & $24(75)$ \\
\hline Ecografía de órbita & $8(25)$ \\
\hline \multicolumn{2}{|l|}{ Campimetría preoperatoria n (\%) } \\
\hline Total & $24(75)$ \\
\hline Normal & $20(83,3)$ \\
\hline Anormal & $4(12,5)$ \\
\hline \multicolumn{2}{|l|}{ Campimetría posoperatoria } \\
\hline Total & $32(100)$ \\
\hline Normal & $28(87,5)$ \\
\hline Anormal & $4(12,5)$ \\
\hline \multicolumn{2}{|l|}{ Agudeza visual preoperatoria } \\
\hline$\leq 20 / 50$ & $28(87,5)$ \\
\hline$<20 / 50$ & $4(12,5)$ \\
\hline \multicolumn{2}{|l|}{ Agudeza visual posperatoria } \\
\hline$\leq 20 / 50$ & $2(6,25)$ \\
\hline$<20 / 50$ & $30(93,75)$ \\
\hline $\begin{array}{l}\text { Exoftalmometría preoperatoria mediana } \mathrm{mm} \\
\text { (RIQ) }\end{array}$ & $24 \mathrm{~mm}(21,8-27)(2,77)$ \\
\hline $\begin{array}{l}\text { Exoftalmometría posoperatoria mediana } \\
m m(R I Q)\end{array}$ & $18,25(16-21)(2,99)$ \\
\hline Neuropatía óptica preoperatoria n (\%) & $2(6,25)$ \\
\hline Neuropatía óptica posoperatoria n (\%) & $0(0)$ \\
\hline \multicolumn{2}{|l|}{ Complicaciones* } \\
\hline Sí & $3(18,75)$ \\
\hline No & $13(81,25)$ \\
\hline
\end{tabular}

* La única complicación encontrada en el estudio fue diplopía.

\section{Discusión}

La orbitopatía distiroidea es la manifestación extratiroidea más común de la enfermedad de Graves ${ }^{(4)}$, caracterizándose por exoftalmos secundario a un incremento en el volumen del tejido orbitario, lo que conlleva con frecuencia a queratitis por exposición, diplopía, neuropatía óptica y alteración estética.

Figura 3. Box Plot comparativo de exoftalmometría prequirúrgica y posquirúrgica en el último control de pacientes con orbitopatía distiroidea por enfermedad de Graves sometidos a descompresión orbitaria endoscópica.

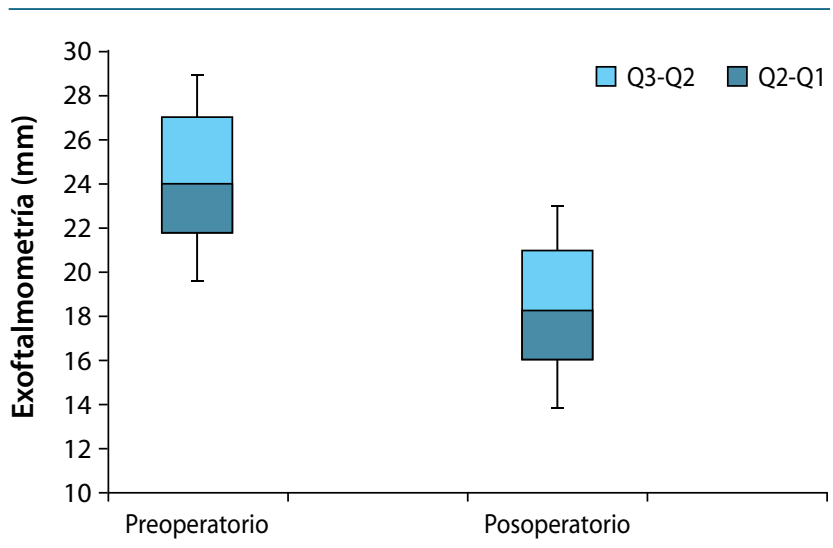


El tratamiento de esta entidad con corticoesteroides sistémicos o radioterapia conlleva usualmente efectos secundarios, por lo cual, la cirugía sigue siendo una de las mejores opciones en el manejo de la orbitopatía por Graves ${ }^{(12)}$.

Con el creciente conocimiento de los procedimientos quirúrgicos disponibles y una mayor experiencia por parte de los cirujanos, el tratamiento exitoso del exoftalmos se ha hecho posible, cada día con menores tasas de complicaciones ${ }^{(16,17)}$.

De la serie de casos presentada se evidenciaron características sociodemográficas, como predominancia del sexo femenino, rangos de edad de los pacientes al momento de someterse a manejo quirúrgico y lateralidad del compromiso, similares a otras series de $\operatorname{casos}^{(18)}$. A diferencia de otros estudios y de la evidencia de la relación entre la orbitopatía distiroidea por Graves y el tabaquismo, sólo se encontró esta relación en el $12,5 \%$ de los pacientes.

Se evidenció mejoría en la agudeza visual en 100\% de los casos, de los cuales 93,7\% presentó una agudeza visual posoperatoria mejor de 20/50. Se demostró, a su vez, mejoría en la exoftalmometría, obteniendo un valor para la mediana posoperatoria de la serie dentro de límites normales, cifras similares a las encontradas por Dubin et al., en el 2008 ${ }^{(6)}$.

La diplopía representa la complicación potencial más frecuente de una descompresión orbitaria por orbitopatía distiroidea y las series de casos muestran una incidencia que varía entre $15 \%$ y $63 \%$ de los pacientes ${ }^{(18)}$, encontrando que los datos en nuestra serie de casos se presentan dentro de los rangos establecidos previamente por la literatura disponible.
Las complicaciones nasosinusales después de una descompresión de órbita vía endoscópica son infrecuentes, sin embargo, se reportan casos de hemorragia posoperatoria, sinequias y sinusitis posoperatoria relacionadas con la obstrucción del drenaje paranasal(19).

Ya que los otorrinolaringólogos rutinariamente advierten a los pacientes no sonarse ni realizar maniobras de Valsalva en el posoperatorio, el enfisema subconjuntival es una complicación poco frecuente. Cuando un paciente lo presenta, el tratamiento es típicamente conservador y presenta una resolución espontánea ${ }^{(20)}$.

\section{Conclusiones}

Consideramos que existen limitaciones en nuestro estudio al ser retrospectivo y descriptivo, ya que supone imprecisión en los criterios de inclusión y un seguimiento irregular; sin embargo, los datos obtenidos permiten vislumbrar el manejo quirúrgico mediante descompresión orbitaria transnasal para orbitopatía de Graves como una intervención segura y eficaz, con menores tasas de complicación y efectos secundarios, comparado con el tratamiento con corticoesteroides sistémicos o la radioterapia.

En esta serie de casos encontramos que es una intervención segura y eficaz, con una baja tasa de complicaciones y fue eficaz según los resultados oftalmológicos, siendo una alternativa superior comparada con el tratamiento con corticoesteroides o la radioterapia.

\section{Referencias}

1. Leong SC, White PS. Outcomes following surgical decompression for dysthyroid orbitopathy (Graves' disease). Curr Opin Otolaryngol Head Neck Surg. 2010 Feb;18(1):37-43. PubMed PMID: 19940769. eng.

2. Robinson D, Wilcsek G, Sacks R. Orbit and orbital apex. Otolaryngol Clin North Am. 2011 Aug;44(4):903-22, viii. PubMed PMID: 21819879. eng.

3. Bartalena L. Graves' orbitopathy: imperfect treatments for a rare disease. Eur Thyroid J. 2013 Dec;2(4):259-69. PubMed PMID: 24783057. PMCID: PMC3923600. eng.

4. Kacker A, Kazim M, Murphy M, Trokel S, Close LG. "Balanced" orbital decompression for severe Graves' orbitopathy: technique with treatment algorithm. Otolaryngol Head Neck Surg. 2003 Feb;128(2):228-35. PubMed PMID: 12601319. eng.

5. Reddy SV, Jain A, Yadav SB, Sharma K, Bhatia E. Prevalence of Graves' ophthalmopathy in patients with Graves' disease presenting to a referral centre in north India. Indian J Med Res. 2014 Jan;139(1):99-104. PubMed PMID: 24604044. PMCID: PMC3994747. eng.

6. Dubin MR, Tabaee A, Scruggs JT, Kazim M, Close LG. Image-guided endoscopic orbital decompression for Graves' orbitopathy. Ann Otol Rhinol Laryngol. 2008 Mar;117(3):177-85. PubMed PMID: 18444477. eng.

7. Metson R, Pletcher SD. Endoscopic orbital and optic nerve decompression. Otolaryngol Clin North Am. 2006 Jun;39(3):551-61, ix. PubMed PMID: 16757231. eng.

8. Vaseghi M, Tarin TT, Levin PS, Terris DJ. Minimally invasive orbital decompression for Graves' ophthalmopathy. Ann Otol Rhinol Laryngol. 2003 Jan;112(1):57-62. PubMed PMID: 12537060. eng.

9. Bijlsma WR, Mourits MP. Radiologic measurement of extraocular muscle volumes in patients with Graves' orbitopathy: a review and guideline. Orbit. 2006 Jun;25(2):83-91. PubMed PMID: 16754214. eng.

10. Daubner D, Spieth S, Engellandt K, von Kummer R. [Diagnosis and differential diagnosis of Graves' orbitopathy in MRI]. Radiologe. 2012 Jun;52(6):550-9. PubMed PMID: 22622414. ger.

11. She YY, Chi CC, Chu ST. Transnasal endoscopic orbital decompression: 15Year clinical experience in Southern Taiwan. J Formos Med Assoc. 2012 Sep. PubMed PMID: 24704350. ENG.

12. Kasperbauer JL, Hinkley L. Endoscopic orbital decompression for Graves ophthalmopathy. Am J Rhinol. 20052005 Nov-Dec;19(6):603-6. PubMed PMID: 16402649. eng.

13. Leung MK, Platt MP, Metson R. Revision endoscopic orbital decompression in the management of Graves' orbitopathy. Otolaryngol Head Neck Surg. 2009 Jul;141(1):46-51. PubMed PMID: 19559957. eng.

14. Lal P, Thakar A, Tandon N. Endoscopic orbital decompression for Graves' orbitopathy. Indian J Endocrinol Metab. 2013 Mar;17(2):265-70. PubMed PMID: 23776900. PMCID: PMC3683202. eng.

15. Eckstein A, Berchner-Pfannschmidt U, Führer D, Esser J. [Update on endocrine orbitopathy]. Ophthalmologe. 2013 Nov;110(11):1079-96. PubMed PMID: 24231915. Ger.

16. Chhabra N, Wu AW, Fay A, Metson R. Endoscopic resection of orbital hemangiomas. Int Forum Allergy Rhinol. 2014 Mar;4(3):251-5. PubMed PMID: 24415525. eng.

17. Cansiz H, Tuskan K, Kalekoğlu N, Oztürk O, Inci E, Pazarli H, et al. [Orbital decompression by endoscopic transnasal and transantral approach in thyroid-associated ophthalmopathy]. Kulak Burun Bogaz Ihtis Derg. 2002 2002 Sep-Oct;9(5):358-62. PubMed PMID: 12471283. tur.

18. Graham SM, Brown CL, Carter KD, Song A, Nerad JA. Medial and lateral orbital wall surgery for balanced decompression in thyroid eye disease. Laryngoscope. 2003 Jul;113(7):1206-9. PubMed PMID: 12838020. eng.

19. Antisdel JL, Gumber D, Holmes J, Sindwani R. Management of sinonasal complications after endoscopic orbital decompression for Graves' orbitopathy. Laryngoscope. 2013 Sep;123(9):2094-8. PubMed PMID: 23839980. eng.

20. Hsu NM, Close LG. Subconjunctival emphysema after orbital decompression for Graves' ophthalmopathy. Otolaryngol Head Neck Surg. 2013 Mar;148(3):523-4. PubMed PMID: 23300222. eng. 